\title{
Childhood Aftention-Deficit/Hyperactivity Disorder Symptoms Are Risk Factors for Obesity and Physical Inactivity in Adolescence
}

\author{
Natasha Khalife, MSc, Marko Kantomaa, PhD, Vivette Glover, PhD, \\ Tuija Tammelin, PhD, Jaana Laitinen, PhD, Hanna Ebeling, MD, \\ Tuula Hurtig, PhD, Marjo-Riitta Jarvelin, MD, PhD, Alina Rodriguez, PhD
}

\begin{abstract}
Objective: To prospectively investigate the association and directionality between attentiondeficit/hyperactivity disorder (ADHD) symptoms and obesity from childhood to adolescence in the general population. We examined whether obesogenic behaviors, namely, physical inactivity and binge eating, underlie the potential ADHD symptom-obesity association. We explored whether childhood conduct disorder (CD) symptoms are related to adolescent obesity/physical inactivity. Method: At 7 to 8 years $(n=8,106)$, teachers reported ADHD and CD symptoms, and parents reported body mass index (BMI) and physically active play. At 16 years $(n=6,934)$, parents reported ADHD symptoms; adolescents reported physical activity (transformed to metabolic equivalent of task [MET] hours per week) and binge eating; BMI and waist-hip ratio (WHR) were measured via clinical examination. Obesity was defined using the International Obesity Task Force (IOTF) cut-offs for BMI and the 95th percentile cut-off for WHR. Results: Childhood ADHD symptoms significantly predicted adolescent obesity, rather than the opposite. Inattention-hyperactivity symptoms at 8 years were associated with indices of obesity at 16 years (obese BMI: odds ratio $[\mathrm{OR}]=1.91,95 \%$ confidence interval $[\mathrm{CI}]=1.10-3.33$; 95th percentile WHR: $\mathrm{OR}=1.71,95 \% \mathrm{CI}=1.05-2.78)$, adjusted for gender, baseline BMI, physical activity, family structure change, and maternal education. Child CD symptoms associated with indices of adolescent obesity. Reduced physically active play in childhood predicted adolescent inattention $(\mathrm{OR}=1.61,95 \% \mathrm{CI}=1.16-2.24)$. Childhood ADHD and CD symptoms were linked with physical inactivity in adolescence (inattentionhyperactivity; $\mathrm{OR}=1.60,95 \% \mathrm{CI}=1.20-2.13$ ), but not binge eating. Physical inactivity mediated the associations. Conclusions: Children with ADHD or CD symptoms are at increased risk for becoming obese and physically inactive adolescents. Physical activity may be beneficial for both behavior problems and obesity. J. Am. Acad. Child Adolesc. Psychiatry, 2014;53(4):425-436. Key Words: attention-deficit/hyperactivity disorder symptoms, conduct disorder symptoms, disruptive behavior, obesity, physical inactivity
\end{abstract}

A growing number of studies report an association between obesity and attentiondeficit/hyperactivity disorder (ADHD).$^{1-4}$ According to a recent review, most studies are limited by use of clinical and/or cross-sectional data. ${ }^{1}$ Furthermore, it is unclear whether the association is specific to ADHD, that is, independent of other behavioral disorders-in particular,

CG Clinical guidance is available at the end of this article.

CME This article can be used to obtain continuing medical education (CME) at www. jaacap.org conduct disorder (CD), ${ }^{5-8}$ one of the primary psychiatric comorbidities of ADHD. ${ }^{9}$ Importantly, studies have not examined potential underlying mechanisms or the direction of the ADHDobesity association. ${ }^{1}$ Research has mainly examined ADHD as a risk factor for obesity. ${ }^{10,11}$ However, it is plausible that the association may be driven from the direction of obesity to ADHD, as factors linked with obesity, for example, leptin (an appetite-regulation hormone) and a sugar-rich diet may contribute to ADHD-like behaviors. ${ }^{12,13}$ Moreover, the association may be bi-directional because of a third underlying factor associated with both obesity and ADHD. ${ }^{14-17}$ Although it Open access under CC BY-NC-ND license. 
is impossible to fully decipher the direction of the association in human beings, prospective longitudinal and epidemiological studies can provide valuable information concerning the general population. Because of the lack of such studies, the direction of the potential link remains unevaluated.

Recently, Cortese et al. reported a long-term link between childhood ADHD and adult obesity, albeit in a small clinical sample $(\mathrm{n}=207)$ of men only. ${ }^{7}$ The same authors, using data from the National Epidemiological Study on Alcohol and Related Conditions, found a significant association, but only for women (retrospective reports for ADHD and self-reports for weight/ height). ${ }^{18}$ These discrepant findings may be attributed to differences in study design, including possible referral bias in the clinical versus population samples. It is necessary to prospectively examine the early developmental trajectory of the potential ADHD-obesity link from childhood to adolescence, because these disorders manifest early in life. ${ }^{19,20}$ The few longitudinal studies of children/adolescents provide inconsistent results, are limited by small sample size, and do not examine directionality. ${ }^{21-23}$ One study in toddlers (i.e., 2-year-olds) reported a link between behavioral difficulties and obesity over a 3.5-year period $^{21}$; however, other studies in older children have not confirmed the association. ${ }^{22,23}$ Another limitation is the sole use of body mass index $(\mathrm{BMI}){ }^{21,22}$ which may not adequately reflect adiposity. ${ }^{24}$ Indicators of abdominal obesity, such as waist-hip ratio (WHR), provide additional measures of adiposity, and may more accurately identify individuals at risk for health consequences of overweight. $^{24}$

Inattention and/or impulsivity symptoms may confer a risk for certain obesogenic behaviors, including physical inactivity and binge eating. ${ }^{1}$ The impact of hyperactivity symptoms remains unclear. ${ }^{1}$ To date, only a few population studies have examined the association between ADHD and physical inactivity in children and/or adolescents, and have yielded conflicting results. ${ }^{25-27}$ There are a few small clinical studies reporting improved behavior in children diagnosed with ADHD after a short-term period or acute bout of physical activity. ${ }^{28-30}$ However, the long-term impact of physical activity on behavior is still unevaluated. Some evidence suggests an association between binge eating and behavioral impulsivity. ${ }^{31,32}$ A recent study found that symptoms of ADHD were significantly, but not differentially, greater in obese patients with and without binge eating behaviors. ${ }^{33}$ Therefore, the extent to which obesogenic behaviors underlie the ADHD-obesity association is unclear.

In this study, we investigated the association and directionality between core ADHD symptoms and obesity (BMI and WHR), from childhood to adolescence, in a large population cohort. We also examined the bi-directional associations between ADHD symptoms and physical activity, and the impact of ADHD symptoms on binge eating, from childhood to adolescence. We hypothesized that there would be a significant association between ADHD symptoms (at a clinically relevant level) and obesity from childhood to adolescence, and that physical inactivity and/or binge eating would mediate this link. Finally, we explored whether CD symptoms, commonly associated with ADHD, are related to obesity, and whether the ADHD symptomobesity link is independent of CD.

\section{METHOD}

\section{Participants}

Participants came from the Northern Finland Birth Cohort (NFBC) 1986. Children with an expected date of birth between July 1, 1985 and June 30, 1986 were eligible; $99 \%$ ( $N=8,954$ live-born singletons) participated. The ethics committee of Northern Ostrobotnia Hospital District approved the study, and both parents and adolescents gave written informed consent.

Postal questionnaires, which included a wide range of health- and lifestyle-related questions, were sent to parents, teachers, and adolescents. At 7 to 8 years $(\mathrm{n}=$ $8,106 ; 91 \%)$, data were gathered via parental and teacher reports. At 16 years $(n=6,934 ; 77 \%)$, data were gathered via parental reports and adolescent selfreports. In addition, adolescents $(n=6,156)$ attended health examinations, which included growth measurements. The NFBC 1986 is a stimulant-naive population sample, ${ }^{34}$ with the exception of 1 boy who received methylphenidate (Ritalin) for a few months at age 12 years to treat ADHD.

\footnotetext{
Measures

Behavior. Teachers assessed child behavior of 8-yearolds using the Rutter B2 scale, ${ }^{35}$ a well-validated screener for childhood mental health. Each of the 26 items is rated as it "certainly applies" (scored 2), "applies somewhat" (scored 1), or "does not apply" (scored 0 ), yielding a total score between 0 and 52. Screening as "probable combined inattention-hyperactivity disorder" is defined as a total score of $\geq 9$ and sum of all 3 inattention-hyperactivity items $\geq 3$. $^{36} \mathrm{We}$ also examined the core ADHD symptoms individually, that is,
} 
inattention (item "poor concentration" $\geq 2$ ) and hyperactivity (sum of items "restless" and "squirmy and fidgety" $\geq 3$ ). CD is defined by a total score of $\geq 9$ and a higher score on the conduct sub-score (sum of 6 items: "destructive," "fights," "is disobedient," "lies," "steals," and "bullies") versus neurotic subscore (sum of 4 items: "often worried," "miserable," "fearful," and "tears on arrival at school").

Parents reported adolescent behavior of 16-yearolds using the Strengths and Weaknesses of ADHD symptoms and Normal behavior (SWAN) scale. ${ }^{37}$ The SWAN is an 18-item scale based on ADHD symptoms listed in the DSM-IV (9 items in the inattention subscale and 9 items in the hyperactive-impulsivity subscale, and together the 18 items indicate ADHD combined subtype). As this scale measures both weaknesses (scored 3, 2, and 1) and strengths (scored -1, -2, and -3 ), along with average behavior (scored 0 ), it is expected to produce a normal distribution of behavioral scores, thereby reducing the risk of overidentifying youths as screening positive for ADHD. The 95th percentile of the distribution of mean scores on each subscale was used as a cut-off point to identify adolescents with ADHD symptoms as probable clinical cases. The cut-off values for the inattention, hyperactive-impulsivity, and combined subscales were, respectively, $0.625,0.125$, and $0.277 .^{34}$

Body Mass Index. Body mass index (BMI) was calculated as weight divided by squared height $\left(\mathrm{kg} / \mathrm{m}^{2}\right)$. At 7 years, parents reported child weight and height. At 16 years, weight and height were measured during a health examination (by trained staff) and were also self-reported. Self-reported height and weight data ( $\mathrm{n}=701 ; 10 \%$ ) were used for those who did not attend the health examination (correlations between clinical and self-report data: height, $\mathrm{r}=0.85, p<.001$; weight, $\mathrm{r}=0.95, p<.001)$. Obesity was defined using the International Obesity Task Force (IOTF) age- and sexspecific cut-off points for BMI. ${ }^{38}$ According to the IOTF cut-off points, BMI was categorized into 3 groups: normal weight, overweight, and obese, at 7 and 16 years.

Waist-Hip Ratio. At 16 years, waist and hip girths were measured at health examinations. Waist-hip ratio (WHR) was calculated as waist girth (in centimeters) divided by hip girth (in centimeters), and dichotomized into normal and abdominal obesity categories, according to the 95th percentile sex-specific cut-off points ( 0.914 for boys and 0.862 for girls). The majority of adolescents in the cohort had reached puberty by 16 years $(n=91 \%)$, and our descriptive analyses showed no significant impact of puberty on the associations under study. Thus, we did not consider puberty further.

Physical Activity. At 7 years, children's preference for physically active play was assessed by asking parents, "Does your child like to participate in active play?" reported as often, sometimes, or hardly ever. The number of children falling into the "hardly ever" category was very small $(0.3 \%)$. Thus, we categorized physically active play into 2 groups: high ("often") and low ("sometimes" and "hardly ever") preference for physically active play.

At 16 years, physical activity outside school hours was evaluated separately for moderate-to-vigorous physical activity and light physical activity by asking the adolescents, "How many hours per week do you participate in brisk activity and light physical activity outside school hours?" In the questionnaire, the term "brisk" was defined as physical activity causing at least some sweating and shortness of breath, while the term "light physical activity" was defined as causing no sweating or shortness of breath. Adolescents also reported their daily time spent in a physically active commute to and from school. The response alternatives (not at all, less than 20 minutes, 20-39 minutes, 40-59 minutes, and at least 1 hour per day) were multiplied by 5 (for 5 school days a week) to correspond to 0,1 , $2.5,3.75$, and 5 hours per week. ${ }^{39}$ The level of physical activity was defined as metabolic equivalent of task (MET)-hours per week, based on the intensity and volume of physical activity, and was divided into quintiles. A MET intensity value of 3 METs was used for light physical activity, 5 METs for brisk physical activity, and 4 METs for commuting physical activity. ${ }^{40}$ The intraclass correlation coefficient for physical activity levels described in terms of quintile categories of MET hours per week was $0.70(95 \% \mathrm{CI}=0.58-0.80)$, and the proportion of subjects who were classified in exactly the same category or next to the same category in 2 different tests was $86 \%$. Adolescents were categorized into 3 groups according to their weekly level of physical activity: active ( 2 highest quintiles), moderately active (third and fourth quintiles), and inactive (lowest quintile). The test-retest reliability of these physical activity questions among a separate group of Finnish adolescents aged 15 to 16 years has been reported to be good. ${ }^{39}$

Binge Eating. At 16 years, an index of binge eating was ascertained by asking adolescents, "How often do you devour large amounts of food?" Binge eating was dichotomized into yes (once a month/once a week/2 or 3 times a week/daily) vs. no (never/hardly ever/ occasionally). This classification was established a priori in the absence of a measure for "loss of control" (essential in defining binge eating), and aims to discriminate more common, occasional episodes of overeating from real binges.

\section{Confounders}

We controlled for potential confounders as indicated by previous research and our descriptive analyses. Gender is associated with both $\mathrm{BMI}^{41}$ and ADHD. ${ }^{42}$ Family structure change indicates change in family structure over time (from 0 to 8 years or from 8 to 16 years), and is important in this study, in which we examined longitudinal associations. The family 
environment has previously been associated with both offspring $\mathrm{BMI}^{43}$ and ADHD. ${ }^{44}$ Family structure change was classified into 4 categories: always a 2-parent family, single-parent family, reconstructed family, and always a 1-parent family. Maternal education, which is a marker of socioeconomic position, has previously been associated with both offspring $\mathrm{BMI}^{45}$ and ADHD. ${ }^{46}$ Educational level categories were defined by the International Standard Classification of Education and the Finnish Board of Education, forming 4 educational categories: basic education ( $\leq 9$ years), upper secondary education (10-12 years), tertiary education ( $\geq 13$ years), and other or degree not finished. BMI and physical activity are closely related, ${ }^{47}$ so we controlled reciprocally for BMI and physical activity as appropriate, to study the association between ADHD symptoms and these 2 variables, independent of one another. Baseline BMI/ADHD symptoms/physically active play were controlled for to allow prediction of "new" cases of obesity, ADHD symptoms, or physical inactivity.

\section{Data Analysis}

Descriptive analyses included frequency distributions for the child/adolescent characteristics and linear regression analyses to examine the associations using continuous measures. At 8 years, baseline associations were examined between ADHD symptoms (combined inattention-hyperactivity, inattention, and hyperactivity)/CD symptoms, BMI, and physically active play. Furthermore, correlation analyses were used to examine comorbidity between inattentionhyperactivity and CD symptoms, as well as associations over the 8-year period.

The main analyses included all variables as categorical measures to indicate clinical levels of concern. ADHD symptoms were examined as screen positive or screen negative (yes/no for probable clinical diagnosis). BMI was categorized as obese/overweight/ normal weight; WHR as abdominal obesity/normal; preference for physically active play as low/high; physical activity as inactive/moderately active/active; and binge eating as yes/no.

We used logistic regression analyses to investigate the longitudinal associations. We assessed ADHD symptoms at 8 years (predictor: teacher Rutter B2) on obesity (BMI and WHR), and physical activity and binge eating at 16 years (outcomes). Likewise, we examined the reverse association by assessing BMI and physically active play at 8 years (predictors) on ADHD symptoms at 16 years (outcome: parent SWAN).

To explore whether the associations under investigation were independent of $C D$, we re-ran the analyses, first, with CD instead of ADHD symptoms and second, with the inclusion only of children who screened positive for either ADHD symptoms or CD symptoms (i.e., "pure" cases). Because of the lack of data on CD in adolescence, we were able to examine only whether CD symptoms predicted obesity, physical inactivity, and binge eating (not vice versa).

We used the bootstrap method m $^{48,49}$ to evaluate whether physical activity and/or binge eating (16 years) mediated the possible association between ADHD symptoms (8 years) and obesity (16 years). Likewise, we examined mediation of the potential CD symptomobesity association by physical activity/binge eating. Mediation confidence intervals were estimated for adjusted analyses, based on 5,000 bootstrap samples.

For all analyses, we controlled for gender, family structure change, current maternal education, BMI/ physical activity (depending on the model), and baseline BMI/ADHD symptoms/physically active play (depending on the model). Analyses were performed using SPSS software, version 20.0. The significance level was defined as $p<.05$.

\section{RESULTS}

Table 1 shows the descriptive statistics of the child/adolescent characteristics. The prevalence of ADHD symptoms at a level indicating clinical relevance in children and adolescents, respectively, were $9.4 \%$ and $5.3 \%$ for combined inattention-hyperactivity; $3.9 \%$ and $5.1 \%$ for inattention symptoms; and $7.1 \%$ and $4.7 \%$ for hyperactivity symptoms. The prevalence of CD symptoms in children was $9.0 \%$. The baseline analyses at 8 years did not show any significant associations between ADHD or CD symptoms and obesity/physically active play.

As expected, descriptive analyses showed high comorbidity between inattention-hyperactivity and $\mathrm{CD}$ symptoms (Pearson $\mathrm{r}=0.67, p<.001 ; \mathrm{n}=$ $570(7.1 \%)$ screening positive for both disorders). Furthermore, correlation analyses demonstrated that the variables of interest (continuous) were significantly associated over time, that is, from 8 to 16 years, for BMI (Pearson $r=0.62, p<.001$ ), inattention-hyperactivity symptoms (Pearson $\mathrm{r}=$ $0.25, p<.001)$, and physically active play/physical activity (Spearman $r=0.12, p<.001$ ). At 16 years, there was a slight negative association between BMI and physical activity (Pearson $\mathrm{r}=-0.04, p<$ $.01)$. BMI and binge eating were related at 16 years (Spearman $\mathrm{r}=0.06, p<.001$ ).

The main aim of the study was to examine the association and directionality between ADHD symptoms and obesity, from childhood to adolescence, as well as mediation by physical activity and binge eating. Adjusted linear regression analyses using continuous measures showed significant longitudinal associations from 8 to 16 years: for example, inattention-hyperactivity symptoms and BMI $(\beta=0.08, p<.001)$, inattention-hyperactivity 
TABLE 1 Characteristics of Children at 8 Years $(n=8,106)$ and Adolescents at 16 Years $(n=6,934)$ in the Northern Finland Birth Cohort, 1986

\begin{tabular}{|c|c|c|c|c|}
\hline \multirow[b]{2}{*}{ Characteristic } & \multicolumn{2}{|c|}{8 Years } & \multicolumn{2}{|c|}{16 Years } \\
\hline & $\mathbf{n}$ & $\%$ & $\mathbf{n}$ & $\%$ \\
\hline \multicolumn{5}{|l|}{ Body weight defined by $\mathrm{BMI}^{\mathrm{a}}$} \\
\hline Normal & 5,758 & 82.7 & 5,667 & 84.7 \\
\hline Overweight & 916 & 13.2 & 787 & 11.8 \\
\hline Obese & 288 & 4.1 & 238 & 3.6 \\
\hline \multicolumn{5}{|l|}{ Abdominal obesity defined by $W H R^{b}$} \\
\hline No & & & 6,109 & 95.0 \\
\hline Yes & & & 320 & 5.0 \\
\hline \multicolumn{5}{|l|}{ Physically active play } \\
\hline Often & 5,954 & 82.1 & & \\
\hline Sometimes & 1,278 & 17.6 & & \\
\hline Hardly ever & 24 & 0.3 & & \\
\hline \multicolumn{5}{|l|}{ Physical activity $-M^{\prime} T^{c}$} \\
\hline Active & & & 2,534 & 40.4 \\
\hline Moderately active & & & 2,486 & 39.6 \\
\hline Inactive & & & 1,258 & 20.0 \\
\hline \multicolumn{5}{|l|}{ Binge eating $^{d}$} \\
\hline No & & & 6,139 & 90.2 \\
\hline Yes & & & 665 & 9.8 \\
\hline \multicolumn{5}{|l|}{ ADHD symptoms (positive diagnostic screene) } \\
\hline Inattention-hyperactivity & 756 & 9.4 & 349 & 5.3 \\
\hline Inattention & 315 & 3.9 & 332 & 5.1 \\
\hline Hyperactivity & 575 & 7.1 & 306 & 4.7 \\
\hline$C D$ symptoms (positive diagnostic screen ${ }^{f}$ ) & 725 & 9.0 & & \\
\hline \multicolumn{5}{|c|}{ "Pure" ADHD symptoms (positive diagnostic screen"), excluding CD cases } \\
\hline Inattention-hyperactivity & 186 & 2.5 & & \\
\hline Inattention & 100 & 1.4 & & \\
\hline Hyperactivity & 184 & 2.5 & & \\
\hline $\begin{array}{l}\text { "Pure" CD symptoms (positive diagnostic screen }{ }^{\dagger} \text { ), excluding } \\
\text { inattention-hyperactivity cases }\end{array}$ & 155 & 2.1 & & \\
\hline \multicolumn{5}{|l|}{ Gender } \\
\hline Male & 4,125 & 50.9 & 3,336 & 48.1 \\
\hline Female & 3,981 & 49.1 & 3,598 & 51.9 \\
\hline \multicolumn{5}{|l|}{ Family structure change (from $0-8$ y or $8-16$ y) } \\
\hline Always a 2-parent family & 6,761 & 85.3 & 4,506 & 75.7 \\
\hline Single-parent family & 513 & 6.5 & 805 & 13.5 \\
\hline Reconstructed family & 537 & 6.8 & 583 & 9.8 \\
\hline Always a single-parent family & 114 & 1.4 & 56 & 0.9 \\
\hline \multicolumn{5}{|l|}{ Maternal education } \\
\hline Basic education ( $\leq 9$ y) & 1,330 & 16.9 & 794 & 13.0 \\
\hline Upper secondary education $(10-12$ y) & 4,318 & 54.9 & 4,045 & 66.4 \\
\hline Tertiary education ( $\geq 13 \mathrm{y})$ & 2,224 & 28.3 & 573 & 9.4 \\
\hline Other or degree not finished & & & 682 & 11.2 \\
\hline \multicolumn{5}{|c|}{ 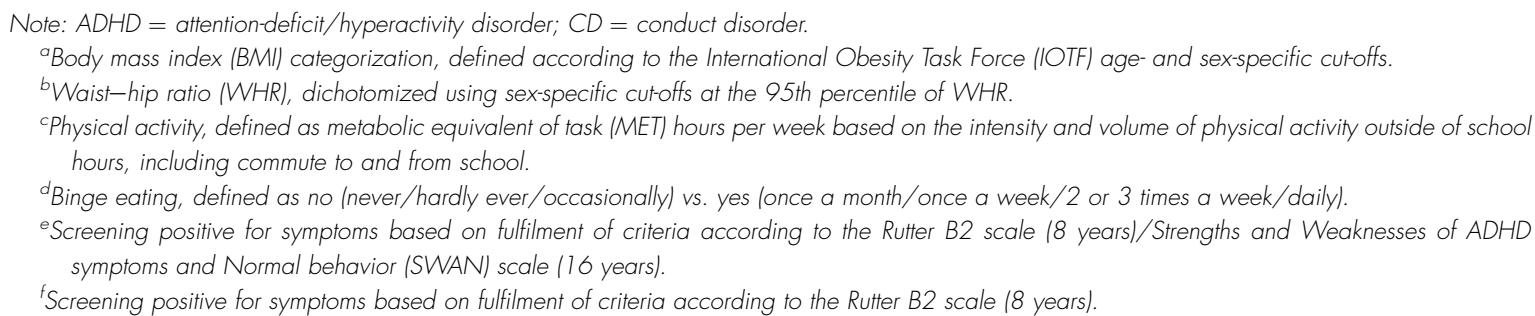 } \\
\hline
\end{tabular}


symptoms and physical activity (MET) $(\beta=-0.04$, $p=.02)$, and physically active play and inattention symptoms $(\beta=0.04, p=.005)$.

The main analyses tested the longitudinal associations using categorical variables, which indicate clinical relevance. The unadjusted logistic regression analyses showed a significant association between probable ADHD at 8 years and obesity at 16 years $(\mathrm{OR}=2.01,95 \% \mathrm{CI}=1.37$ 3.00 ), but nonsignificance in the opposite direction, that is, from obesity at 8 years to probable ADHD at 16 years (OR $=0.90,95 \%$ CI $=0.69$ 1.18). There were significant associations between probable ADHD at 8 years and physical inactivity at 16 years $(\mathrm{OR}=1.30,95 \% \mathrm{CI}=1.01-1.67)$, and reduced physically active play at 8 years and inattention at 16 years $(\mathrm{OR}=1.53,95 \% \mathrm{CI}=$ 1.15-2.05).

The adjusted analyses revealed similar results. Probable ADHD at 8 years predicted obesity and abdominal obesity at 16 years (Table 2). Screening positive for either inattention or hyperactivity predicted abdominal obesity by more than 2 -fold; inattention also predicted overweight and obesity (Table 2). There was no evidence for the reverse association; that is, obesity did not predict ADHD symptoms over the long term (Table 3). Low preference for physically active play at 8 years was associated with inattention symptoms at 16 years (Table 3). There was evidence for the reverse association, as inattention-hyperactivity and inattention symptoms were longitudinally associated with physical inactivity (Table 4). We did not find any significant longitudinal associations between ADHD symptoms and binge eating (Table 4).

We explored whether the associations under study were independent of CD symptoms. Screening positive for probable $\mathrm{CD}$ was associated with overweight, obesity, abdominal obesity (Table 2), and physical inactivity, but not binge eating (Table 4). In analyses excluding children with CD symptoms, "pure" inattention and "pure" hyperactivity symptoms at 8 years individually predicted abdominal obesity at 16 years (Table 2 ). In analyses excluding children with inattentionhyperactivity symptoms, "pure" CD symptoms at 8 years predicted overweight at 16 years (Table 2). Neither "pure" ADHD nor "pure" CD symptoms was associated with physical inactivity or binge eating.

Bootstrapping showed that physical inactivity at 16 years mediated the longitudinal association between inattention-hyperactivity symptoms and obesity (bootstrap estimate $=0.004$, percentile $95 \% \mathrm{CI}=0.001-0.007, \mathrm{n}=4,524)$, and CD symptoms and obesity (bootstrap estimate = 0.003 , percentile $95 \% \mathrm{CI}=0.001-0.007, \mathrm{n}=4,514$ ). In contrast, there was no evidence for mediation by binge eating at 16 years (for inattentionhyperactivity: bootstrap estimate $=0.001$, percentile $95 \% \mathrm{CI}=-0.001$ to $0.003, \mathrm{n}=5,056$; for CD: bootstrap estimate $=0.001$, percentile $95 \%$ $\mathrm{CI}=-0.001$ to $0.003, \mathrm{n}=5,044)$.

\section{DISCUSSION}

To our knowledge, we are the first to report that childhood ADHD symptoms prospectively predict adolescent obesity (clinically measured as BMI, defined according to IOTF guidelines), and abdominal obesity (defined according to the $95^{\text {th }}$ percentile for WHR). Childhood ADHD symptoms predicted later physical inactivity (MET) in adolescence. Reduced physically active play in childhood also predicted inattention in adolescence. Thus, we report evidence for a bidirectional association between ADHD symptoms and physical inactivity. Furthermore, we also report an association between child CD symptoms, adolescent obesity (BMI and WHR), and physical inactivity. However, it was unclear whether ADHD and CD symptoms independently predicted these associations.

We focused on the ADHD-obesity link because of the substantial cross-sectional evidence that a comorbid association exists between obesity and ADHD in children and adolescents. ${ }^{1}$ Using a large, longitudinal, medically untreated population cohort, we were able not only to confirm the ADHD symptom-obesity link, but also to address the issue of directionality. Our results showed that the association was driven in the direction of ADHD symptoms to obesity (and not vice versa) over an 8-year period, from childhood to adolescence.

Our longitudinal data provide insight into the pathogenesis underlying the ADHD symptomobesity association. Inattention and hyperactivity were each important in predicting later overweight or obesity, whereas inattention was important in predicting later physical inactivity. Mediation analysis provided evidence for a mediatory effect of physical inactivity on both the ADHD symptom-obesity and the CD symptomobesity associations. It is possible that social impairments (e.g., peer problems and rule breaking) related to ADHD or $\mathrm{CD}$ may hinder child 
TABLE 2 Odds Ratios (OR) and 95\% Cl for the Associations Between Attention-Deficit/Hyperactivity Disorder and Conduct Disorder (CD) Probable Cases at 8 Years and Obesity at 16 Years $^{a}$

\begin{tabular}{|c|c|c|c|c|c|c|c|c|c|c|c|c|c|}
\hline \multirow[b]{3}{*}{ Rutter B2 (Teacher Report) } & \multicolumn{8}{|c|}{ BMI } & \multicolumn{5}{|c|}{ WHR $^{c}$} \\
\hline & \multirow[b]{2}{*}{ n Cases } & \multirow[b]{2}{*}{ n Controls } & \multicolumn{3}{|c|}{ Overweight vs. Normal } & \multicolumn{3}{|c|}{ Obese vs. Normal } & \multirow[b]{2}{*}{$\mathrm{n}$ Cases } & \multirow[b]{2}{*}{ n Controls } & \multicolumn{3}{|c|}{ Abdominal Obesity vs. Normal } \\
\hline & & & OR & $95 \% \mathrm{Cl}$ & $p$ & OR & $95 \% \mathrm{Cl}$ & $p$ & & & OR & $95 \% \mathrm{Cl}$ & $p$ \\
\hline \multicolumn{14}{|l|}{ Screen-positive cases } \\
\hline Inattention-hyperactivity & 332 & 4,192 & 1.32 & $0.93-1.88$ & .13 & 1.91 & $1.10-3.33$ & .02 & 274 & 3,784 & 1.71 & $1.05-2.78$ & $\begin{array}{c}.03 \\
<001\end{array}$ \\
\hline Inattention & 134 & 4,392 & 1.71 & $1.04-2.83$ & .04 & 2.35 & $1.09-5.07$ & .03 & 110 & 3,950 & 3.46 & $1.91-6.26$ & $<.001$ \\
\hline Hyperactivity & 257 & 4,272 & 1.44 & $0.97-2.13$ & .07 & 1.77 & $0.94-3.34$ & .08 & 220 & 3,843 & 2.14 & $1.28-3.59$ & $<.01$ \\
\hline Conduct & 335 & 4,179 & 1.52 & $1.07-2.14$ & .02 & 2.53 & $1.49-4.31$ & $<.01$ & 282 & 3,767 & 1.67 & $1.03-2.71$ & .04 \\
\hline \multicolumn{14}{|l|}{ "Pure" cases } \\
\hline Inattention-hyperactivity ${ }^{d}$ & 78 & 4,100 & 1.34 & $0.66-2.71$ & .42 & 0.75 & $0.16-3.56$ & .71 & 61 & 3,705 & 1.55 & $0.52-4.60$ & .43 \\
\hline Inattention ${ }^{d}$ & 42 & 4,136 & 2.11 & $0.92-4.81$ & .08 & 1.51 & $0.30-7.63$ & .62 & 35 & 3,732 & 6.91 & $2.89-16.53$ & $<.001$ \\
\hline Hyperactivity $^{d}$ & 86 & 4,090 & 1.50 & $0.77-2.91$ & .23 & 2.10 & $0.66-6.70$ & .21 & 79 & 3,685 & 3.83 & $1.82-8.06$ & $<.001$ \\
\hline Conduct $^{e}$ & 81 & 4,100 & 2.13 & $1.14-4.00$ & .02 & 2.75 & $0.96-7.86$ & .06 & 69 & 3,705 & 1.38 & $0.47-4.03$ & .56 \\
\hline \multicolumn{14}{|c|}{ ized by body mass index (BMI) and waist-hip ratio (WHR), and adjusted for gender, body mass index (8 years), physical activity (16 years), family structure change (ffrom 8 to 16 years), and maternal } \\
\hline
\end{tabular}




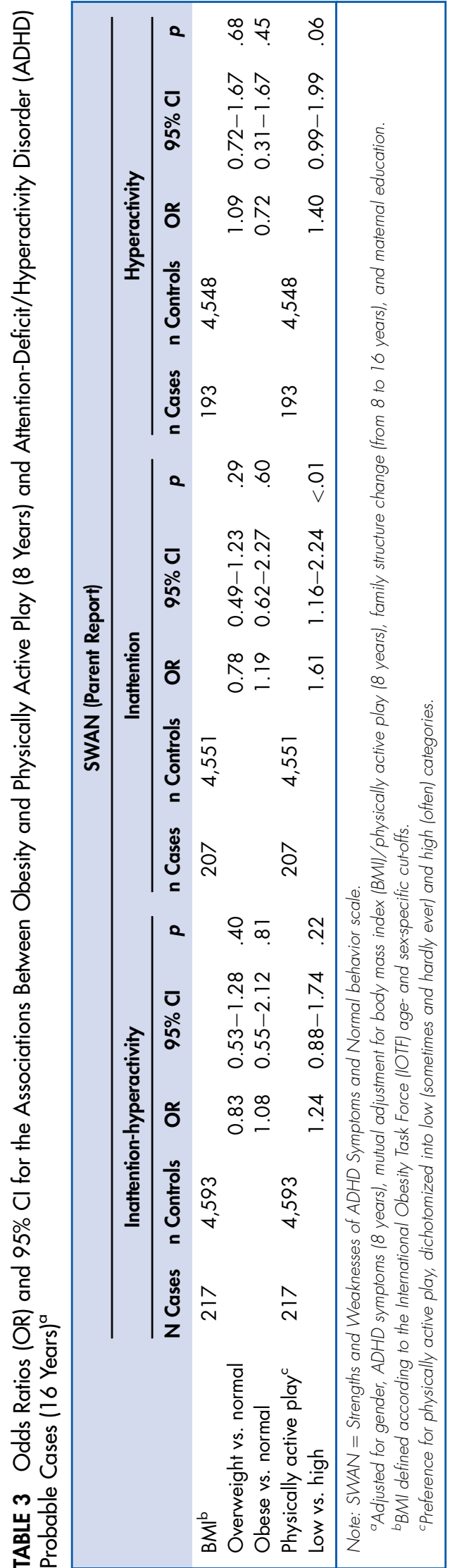

participation in structured physical activities, particularly in organized settings and team activities. Instead of exercising, children with ADHD or CD behaviors may spend more time watching TV and playing video games. ${ }^{50-53}$ This lack of physical activity could contribute to weight gain over time. Our results show that inattention symptoms are driving the ADHD symptom-physical inactivity-obesity pathway. Physical activities require increased concentration, perception, and self-directedness, which may be challenging for children with inattention. There are common biological systems, for example, dopamine and brain-derived neurotrophic factor (BDNF), that are associated with ADHD, ${ }^{14,15}$ obesity, ${ }^{16,17}$ and physical inactivity, ${ }^{54,55}$ which further support the concept of a link among all of these conditions. Less is known about potential mechanisms linking $\mathrm{CD}$ and obesity, and thus this needs further study.

It has been proposed that inattention may induce abnormal eating behaviors, such as binge eating, ${ }^{4,33}$ which in turn may contribute to the development of obesity. However, we did not find support for this idea, as core ADHD symptoms in childhood were not associated with later binge eating in adolescence. Impulsivity has previously been linked with binge eating ${ }^{31,32}$; however, we could not study this association because of a lack of data on impulsivity. Furthermore, it may be possible that ADHD symptoms are associated with other disordered eating patterns that are not measured here. For example, hyperactivity may be linked with obesity via emotionally driven eating, a possible mechanism to cope with the frustration related to the restlessness that accompanies hyperactivity.

Because of the high comorbidity previously reported ${ }^{9}$ and observed in our dataset between ADHD and CD, it is important to consider whether $C D$ accounts for the associations between ADHD and obesity/physical inactivity. Our exploratory analyses using only "pure" cases showed that inattention and hyperactivity symptoms each remained significantly associated with abdominal obesity, and CD symptoms remained associated with overweight. This suggests that "pure" ADHD and CD symptoms predict differential obesity outcomes. These results involving "pure" cases are inconsistent with the original results that showed significant associations across a wider range of obesity/physical activity outcomes. This inconsistency may be due to reduced statistical power or may reflect true etiological 
TABLE 4 Odds Ratios (OR) and 95\% Cl for the Associations Between Attention-Deficit/Hyperactivity Disorder (ADHD) and Conduct Disorder (CD) Probable Cases (8 Years) and Physical Activity and Binge Eating (16 Years) ${ }^{a}$

\begin{tabular}{|c|c|c|c|c|c|c|c|c|c|c|c|c|c|}
\hline \multirow[b]{3}{*}{ Rutter B2 (Teacher Report) } & \multicolumn{8}{|c|}{ Physical Activity ${ }^{b}$} & \multicolumn{5}{|c|}{ Binge Eating $^{c}$} \\
\hline & \multirow[b]{2}{*}{ n Cases } & \multirow[b]{2}{*}{ n Controls } & \multicolumn{3}{|c|}{ Moderately Active vs. Active } & \multicolumn{3}{|c|}{ Inactive vs. Active } & \multirow[b]{2}{*}{ n Cases } & \multirow[b]{2}{*}{ n Controls } & \multicolumn{3}{|c|}{ Yes vs. No } \\
\hline & & & OR & $95 \% \mathrm{Cl}$ & $p$ & OR & $95 \% \mathrm{Cl}$ & $p$ & & & OR & $95 \% \mathrm{Cl}$ & $p$ \\
\hline \multicolumn{14}{|l|}{ Screen-positive cases } \\
\hline Inattention-hyperactivity & 368 & 4,568 & 1.27 & $0.99-1.64$ & .06 & 1.60 & $1.20-2.13$ & $<.01$ & 378 & 4,678 & 1.20 & $0.85-1.68$ & .29 \\
\hline Inattention & 145 & 4,792 & 1.27 & $0.85-1.90$ & .24 & 1.89 & $1.24-2.89$ & $<.01$ & 152 & 4,906 & 0.99 & $0.57-1.71$ & .98 \\
\hline Hyperactivity & 284 & 4,654 & 1.15 & $0.86-1.52$ & .35 & 1.37 & $0.99-1.88$ & .06 & 292 & 4,766 & 0.98 & $0.65-1.47$ & .91 \\
\hline Conduct & 359 & 4,564 & 1.21 & $0.94-1.56$ & .14 & 1.47 & $1.10-1.97$ & $<.01$ & 369 & 4,675 & 1.27 & $0.91-1.78$ & .16 \\
\hline \multicolumn{14}{|l|}{ "Pure" cases } \\
\hline Inattention-hyperactivity $^{d}$ & 90 & 4,474 & 1.19 & $0.73-1.94$ & .49 & 1.50 & $0.87-2.60$ & .15 & 95 & 4,579 & 0.85 & $0.41-1.78$ & .67 \\
\hline Inattention $^{d}$ & 48 & 4,515 & 0.81 & $0.41-1.61$ & .55 & 1.44 & $0.71-2.92$ & .32 & 52 & 4,622 & 0.76 & $0.27-2.14$ & .61 \\
\hline Hyperactivity $^{d}$ & 101 & 4,458 & 1.09 & $0.69-1.74$ & .70 & 1.38 & $0.82-2.32$ & .23 & 104 & 4,565 & 0.97 & $0.50-1.90$ & .94 \\
\hline Conduct $^{\mathrm{e}}$ & 81 & 4,474 & 0.98 & $0.60-1.63$ & .95 & 1.08 & $0.59-1.98$ & .80 & 86 & 4,579 & 1.09 & $0.54-2.20$ & .82 \\
\hline
\end{tabular}

Note: ${ }^{a}$ Adiusted for gender, physically active play at 8 years (for model ${ }^{b}$ ), body mass index (BMIl) at 16 years (for model ${ }^{b}$ ), family structure change (from 8 to 16 years), and maternal education.

bPhysical activity, defined as metabolic equivalent of task (MET) hours per week based on the intensity and volume of physical activity outside of school hours, including commute to and from school.

'Binge eating, defined as yes (once a month/once a week/2 or 3 times a week/daily) vs. no (never/hardly ever/occasionally).

Excluding children screening positive for CD symptoms.

Excluding children screening positive for inattention-hyperactivity symptoms. 
differences; thus, further study is needed. The OR confidence intervals between ADHD and CD symptoms overlap, suggesting that there is no significant difference in the prediction of overweight/obesity by ADHD and CD. It may thus be the case that comorbid ADHD is most important in conferring risk. Individuals with ADHD and comorbid CD experience problems with impulse control and/or reward processing, which may reflect a common genetic basis for behavioral disorders and obesity. ${ }^{8}$ However, it is difficult to draw conclusions from our results because of the small sample sizes of "pure" cases. Larger studies are required to expand on our preliminary findings, as well as to understand the pathways by which each type of behavioral disorder may contribute to obesity, and whether comorbidity confers the greatest risk.

Although previous studies have found that physical exercise-either a short-term training period or an acute bout-improves behavior in children with $\mathrm{ADHD},{ }^{28-30}$ we extend these findings by identifying a long-term link between reduced physically active play and inattention symptoms. These results partly support previous hypotheses that physically active play may be one of the most important factors influencing human development, ${ }^{56}$ and that social play may alleviate ADHD symptoms in children. ${ }^{57}$ Physical inactivity has also been linked with both general mental health and scholastic difficulties in adolescents. ${ }^{58,59}$ Considering the available evidence, it is possible to speculate that regular physical activity may alleviate ADHD symptoms over the long term.

This study has several strengths. First, the longitudinal design allowed us to identify the directionality of the ADHD symptom-obesity association, as well as to study the long-term link between ADHD symptoms and both physical inactivity and binge eating prospectively. Second, we used a population-based sample, which reduces the confounding effects that are often observed in clinical studies, for example, symptom severity, referral, and medication biases. Third, we used 2 indices of obesity measured clinically, namely, BMI and WHR, to provide a more complete measure of adiposity. Fourth, we examined the core ADHD symptoms, which enabled us to determine that both inattention and hyperactivity contribute to the associations. Fifth, by examining "pure" ADHD and CD cases, we explored whether each type of disorder was specifically related to obesity.
There are also several limitations to this study. First, despite the longitudinal design and the attempt to control for important confounders, we cannot affirm a causal link between ADHD or CD symptoms and obesity because of the observational nature of the study. This is an inherent problem in human studies. Second, we could not examine impulsivity symptoms because the Rutter B2 scale, used by teachers to report child behavior, does not measure impulsivity symptoms per se, and the SWAN, used by parents to report adolescent behavior, combines hyperactivity and impulsivity symptoms (reflecting $D S M-I V$ criteria for a hyperactive-impulsive ADHD subtype). Third, we could not examine bi-directionality between CD-obesity/physical activity because of the lack of parental report on adolescent CD. Fourth, we did not account for ADHD psychiatric comorbidities in the analyses beyond $C D$, because of limitations in data availability. Fifth, the participating children's height and weight at 7 years were reported by parents rather than clinically measured. Sixth, MET data were unavailable at 8 years, so instead we used data on preference for "physically active play" to indicate physical activity levels in childhood. Finally, we were unable to assess gender differences, because stratification by sex significantly reduced our statistical power.

Despite these limitations, our findings have significant public health implications, with the potential for novel prevention/treatment strategies for both obesity and ADHD symptoms. Children with ADHD and/or CD behaviors can be monitored for overweight/obesity at an early stage, thus potentially averting a developmental trajectory of obesity. This work provides support for the long-term benefits of regular physical activity on ADHD behaviors, emphasizing the importance of promoting physical education in schools, despite pressure to cut back to allow more time for academic classes. Physical inactivity, ADHD, and CD symptoms have all been linked with poor academic performance, ${ }^{59-61}$ so the promotion of physical education and associated behavioral benefits may actually help to improve academic outcomes, as well as improving other mental and physical health outcomes in the longterm. Randomized controlled trials are desperately needed to examine whether physical activity could be used as a behavioral intervention in the treatment of ADHD and CD.

To summarize, here we have shown that children with ADHD or CD symptoms are at 
increased risk for becoming obese and physically inactive adolescents. We found novel evidence that physical inactivity mediates both the ADHD symptom-obesity and CD symptom-obesity associations. We show that reduced physically active play in childhood constitutes an early risk factor for inattention symptoms later in adolescence. Considering the escalating prevalence of

\section{CG Clinical Guidance}

- This prospective, longitudinal, population-based study found that children with ADHD or CD symptoms are at increased risk for becoming obese and physically inactive adolescents. Clinicians should monitor weight gain in children with $\mathrm{ADHD}$ or $\mathrm{CD}$ symptoms to reduce the risk of later overweight/ obesity.

- Physical inactivity was observed to mediate the association between ADHD symptoms and obesity, and between CD symptoms and obesity. Therefore, physical activity should be encouraged in children and adolescents with ADHD or CD symptoms.

- Physical activity may also alleviate ADHD symptoms in the long term. Low preference for physically active play in childhood was linked with inattention symptoms in adolescence. This suggests that physically active play may have long-term beneficial effects on child behavior. Randomized controlled trials are needed to confirm these findings.

\section{REFERENCES}

1. Cortese S, Vincenzi B. Obesity and ADHD: clinical and neurobiological implications. Curr Topics Behav Neurosci. 2012;9:199-218.

2. Altfas J. Prevalence of attention deficit/hyperactivity disorder among adults in obesity treatment. BMC Psychiatry. 2002;2:9.

3. Agranat-Meged $T$, Deitcher C, Goldzweig G, Leibenson L, Stein M, Galili-Wesstub E. Childhood obesity and attention deficit/hyperactivity disorder: a newly described comorbidity in obese hospitalized children. Int J Eat Disord. 2005;37:357-359.

4. Davis C. Attention-deficit/hyperactivity disorder: associations with overeating and obesity. Curr Psychiatry Rep. 2010;12:389-395.

5. Duarte CS, Sourander A, Nikolakaros G, et al. Child mental health problems and obesity in early adulthood. J Pediatr. 2010;156:93-97.

6. Erhart M, Herpertz-Dahlmann B, Wille N, Sawitzky-Rose B, Hölling H, Ravens-Sieberer U. Examining the relationship between attention-deficit/hyperactivity disorder and overweight in children and adolescents. Eur Child Adolesc Psychiatry. 2012;21:39-49.

7. Cortese S, Ramos Olazagasti MA, Klein RG, Castellanos FX, Proal E, Mannuzza S. Obesity in men with childhood ADHD: a 33-year controlled, prospective, follow-up study. Pediatrics. 2013; 131:1731-1738

8. Pauli-Pott U, Neidhard J, Heinzel-Gutenbrunner M, Becker K. On the link between attention deficit/hyperactivity disorder and obesity: do comorbid oppositional defiant and conduct disorder matter? Eur Child Adolesc Psychiatry. 2013; Nov 7 http:/ /dx.doi. org/10.1007/s00787-013-0489-4.

9. Biederman J, Newcorn J, Sprich S. Comorbidity of attention deficit hyperactivity disorder with conduct, depressive, anxiety, and other disorders. Am J Psychiatry. 1991;148:564-577. both obesity and ADHD/CD, our novel findings are of public health significance, providing insight into additional risk factors and paving the way for new prevention/treatment strategies for these chronic disorders. $\varepsilon$

Accepted January 27, 2014

Ms. Khalife and Drs. Kantomaa, Glover, Jarvelin, and Rodriguez are with Imperial College London, UK. Dr. Kantomaa and Tammelin are with LIKES - Research Center for Sports and Health Sciences, Finland. Dr. Laitinen is with the Finnish Institute for Occupational Health, Finland. Dr. Ebeling is with the Clinic of Child Psychiatry, University and University Hospital of Oulu, Finland. Dr. Hurtig is with the Institute of Health Sciences, University of Oulu, Finland. Dr. Jarvelin also is with the Medical Research Council (MRC) Health Protection Agency (HMA) Centre for Environment and Health, Imperial College, UK; Institute of Health Sciences, University of Oulu, Finland; Biocenter Oulu, University of Oulu, Finland; Unit of Primary Care, Oulu University Hospital, Finland; and the National Institute for Health and Welfare, Finland. Dr. Rodriguez is also with Mid Sweden University, Sweden. This work was funded by the Academy of Finland (103451); Sigrid Juselius Foundation, Finland; Thule Institute, University of Oulu, Finland; the National Institute of Mental Health (MH63706); and Biological, Clinical and Genetic Markers of Future Risk of Cardiovascular Disease (EURO-BLCS). Dr. Rodriguez received funding partly from the Swedish Council for Working Life and Social Research (FAS).

The authors express gratitude to the late Paula Rantakallio, MD, PhD, of the University of Oulu, for the launch of the Northern Finland Birth Cohort in 1986.

Disclosure: Ms. Khalife and Drs. Kantomaa, Glover, Tammelin, Laitinen, Ebeling, Hurtig, Jarvelin, and Rodriguez report no biomedical financial interests or potential conflicts of interest.

Correspondence to Alina Rodriguez, PhD, Imperial College London, St Mary's Campus, London W2 1PG, UK; e-mail: a.rodriguez@ imperial.ac.uk

0890-8567/\$36.00/@2014 American Academy of Child and Adolescent Psychiatry

http://dx.doi.org/10.1016/i.jaac.2014.01.009

10. Korczak DJ, Lipman E, Morrison K, Szatmari P. Are children and adolescents with psychiatric illness at risk for increased future body weight? A systematic review. Dev Med Child Neurol. 2013; 55:980-987.

11. Fuemmeler BF, Ostbye T, Yang C, McClernon FJ, Kollins SH. Association between attention-deficit/hyperactivity disorder symptoms and obesity and hypertension in early adulthood: a population-based study. Int J Obes. 2011;35:852-862.

12. Paz-Filho GJ, Babikian T, Asarnow R, et al. Leptin replacement improves cognitive development. PLoS One. 2008;3:e3098.

13. Johnson R, Gold M, Johnson D, et al. Attention-deficit/hyperactivity disorder: is it time to reappraise the role of sugar consumption? Postgrad Med J. 2011;123:39-49.

14. Comings DE, Blum K. Reward deficiency syndrome: genetic aspects of behavioral disorders. Prog Brain Res. 2000;126:325-341.

15. Kent L, Green E, Hawi Z, et al. Association of the paternally transmitted copy of common Valine allele of the Val66Met polymorphism of the brain-derived neurotrophic factor (BDNF) gene with susceptibility to ADHD. Mol Psychiatry. 2005;10:939-943.

16. Gami AS, Caples SM, Somers VK. Obesity and obstructive sleep apnea. Endocrinol Metab Clin North Am. 2003;32:869-894.

17. Gray J, Yeo GSH, Cox JJ, et al. Hyperphagia, severe obesity, impaired cognitive function, and hyperactivity associated with functional loss of one copy of the brain-derived neurotrophic factor (BDNF) gene. Diabetes. 2006;55:3366-3371.

18. Cortese S, Faraone SV, Bernardi S, Wang S, Blanco C. Adult attention-deficit hyperactivity disorder and obesity: epidemiological study. Br J Psychiatry. 2013;203:24-34. 
19. Klish WJ. Childhood obesity. Pediatr Rev. 1998;19:312-315.

20. Kessler RC, Adler LA, Barkley R, et al. Patterns and predictors of attention-deficit/hyperactivity disorder persistence into adulthood: results from the national comorbidity survey replication. Biol Psychiatry. 2005;57:1442-1451.

21. Graziano PA, Calkins SD, Keane SP. Toddler self-regulation skills predict risk for pediatric obesity. Int J Obes. 2010;34:633-641.

22. Mustillo S, Worthman C, Erkanli A, Keeler G, Angold A, Costello EJ. Obesity and psychiatric disorder: developmental trajectories. Pediatrics. 2003;111:851-859.

23. Biederman J, Spencer TJ, Monuteaux MC, Faraone SV. A naturalistic 10-year prospective study of height and weight in children with attention-deficit hyperactivity disorder grown up: sex and treatment effects. J Pediatr. 2010;157:635-640.

24. Romero-Corral A, Montori VM, Somers VK, et al. Association of bodyweight with total mortality and with cardiovascular events in coronary artery disease: a systematic review of cohort studies. Lancet. 2006;368:666-678.

25. Martikainen S, Pesonen A-K, Lahti J, et al. Physical activity and psychiatric problems in children. J Pediatr. 2012;161: $160-162$.

26. van Egmond-Fröhlich AWA, Weghuber D, de Zwaan M. Association of symptoms of attention-deficit/hyperactivity disorder with physical activity, media time, and food intake in children and adolescents. PLoS One. 2012;7:e49781.

27. Barnard-Brak L, Davis T, Sulak T, Brak V. The association between physical education and symptoms of attention deficit hyperactivity disorder. J Phys Activ Health. 2011;8:964-970.

28. McKune AJ, Pautz J, Lombard J. Behavioural response to excercise in children with attention-deficit/hyperactivity disorder. S Afr J Sports Med. 2003;17-21.

29. Verret C, Guay M-C, Berthiaume C, Gardiner P, Béliveau L. A physical activity program improves behavior and cognitive functions in children with ADHD: an exploratory study. J Atten Disord. 2012;16:71-80.

30. Pontifex MB, Saliba BJ, Raine LB, Picchietti DL, Hillman CH. Exercise improves behavioral, neurocognitive, and scholastic performance in children with attention-deficit/hyperactivity disorder. J Pediatr.162:543-551.

31. Cortese S, Isnard P, Frelut ML, et al. Association between symptoms of attention-deficit/hyperactivity disorder and bulimic behaviors in a clinical sample of severely obese adolescents. Int J Obes. 2007;31:340-346.

32. Kelly NR, Bulik CM, Mazzeo SE. Executive functioning and behavioral impulsivity of young women who binge eat. Int J Eat Disord. 2013;46:127-139.

33. Davis C, Patte K, Levitan RD, et al. A psycho-genetic study of associations between the symptoms of binge eating disorder and those of attention deficit (hyperactivity) disorder. J Psychiatr Res. 2009;43:687-696.

34. Smalley SL, McGough JJ, Moilanen IK, et al. Prevalence and psychiatric comorbidity of attention-deficit/hyperactivity disorder in an adolescent Finnish population. J Am Acad Child Adolesc Psychiatry. 2007:46:1575-1583.

35. Rutter M. A children's behaviour questionnaire for completion by teachers: preliminary findings. J Child Psychol Psychiatry. 1967;8:1-11.

36. Rodriguez A, Kaakinen M, Moilanen I, et al. Mixed-handedness is linked to mental health problems in children and adolescents. Pediatrics. 2010;125:e340-e348.

37. Swanson JM, Schuck S, Mann M, et al. Categorical and dimensional definitions and evaluations of symptoms of ADHD: the SNAP and SWAN ratings scales. Available at: http:/ /www.adhd. net/SNAP_SWAN.pdf. Accessed June 27, 2013.

38. Cole TJ, Bellizzi MC, Flegal KM, Dietz WH. Establishing a standard definition for child overweight and obesity worldwide: international survey. BMJ. 2000;320:1240.
39. Tammelin T, Ekelund U, Remes J, Nayha S. Physical activity and sedentary behaviors among Finnish youth. Med Sci Sports Exerc. 2007;39:1067-1074

40. Ainsworth BE, Haskell WL, Leon AS, et al. Compendium of physical activities: classification of energy costs of human physical activities. Med Sci Sports Exerc. 1993;25:71-80.

41. Storey ML, Forshee RA, Weaver AR, Sansalone WR. Demographic and lifestyle factors associated with body mass index among children and adolescents. Int J Food Sci Nutr. 2003;54:491-503.

42. Faraone SV, Sergeant J, Gillberg C, Biederman J. The worldwide prevalence of ADHD: is it an American condition? World Psychiatry. 2003;2:104-113.

43. Schmeer KK. Family structure and obesity in early childhood. Soc Sci Res. 2012;41:820-832

44. Banerjee TD, Middleton F, Faraone SV. Environmental risk factors for attention-deficit hyperactivity disorder. Acta Pædiatrica. 2007; 96:1269-1274.

45. Chivers P, Parker H, Bulsara M, Beilin L, Hands B. Parental and early childhood influences on adolescent obesity: a longitudinal study. Early Child Dev Care. 2012;182:1071-1087.

46. Hjern A, Weitoft GR, Lindblad F. Social adversity predicts ADHDmedication in school children-a national cohort study. Acta Pædiatrica. 2010;99:920-924.

47. Bauman AE, Reis RS, Sallis JF, Wells JC, Loos RJF, Martin BW. Correlates of physical activity: why are some people physically active and others not? Lancet. 2012;380:258-271.

48. Bollen K, Stine R. Direct and indirect effects: classical and bootstrap estimates of variability. Sociol Methodol. 1990;20:115-140.

49. Preacher K, Hayes A. SPSS and SAS procedures for estimating indirect effects in simple mediation models. Behav Res Methods Instrum Comput. 2004;36:717-731.

50. Waring ME, Lapane KL. Overweight in children and adolescents in relation to attention-deficit/hyperactivity disorder: results from a national sample. Pediatrics. 2008;122:e1-e6.

51. Kim J, Mutyala B, Agiovlasitis S, Fernhall B. Health behaviors and obesity among US children with attention deficit hyperactivity disorder by gender and medication use. Prev Med. 2011;52:218-222.

52. Robertson LA, McAnally HM, Hancox RJ. Childhood and adolescent television viewing and antisocial behavior in early adulthood. Pediatrics. 2013;131:439-446.

53. Kronenberger WG, Mathews VP, Dunn DW, et al. Media violence exposure in aggressive and control adolescents: differences in selfand parent-reported exposure to violence on television and in video games. Aggress Behav. 2005;31:201-216.

54. Tantillo M, Kesick CM, Hynd GW, Dishman RK. The effects of exercise on children with attention-deficit hyperactivity disorder. Med Sci Sports Exerc. 2002;34:203-212.

55. Zoladz JA, Pilc A. The effect of physical activity on the brain derived neurotrophic factor: from animal to human studies. J Physiol Pharmacol. 2010;61:533-541.

56. Pellegrini AD, Smith PK. Physical activity play: the nature and function of a neglected aspect of play. Child Dev. 1998;69:577-598.

57. Panksepp J. Can play diminish ADHD and facilitate the construction of the social brain? J Can Acad Child Adolesc Psychiatry. 2007;16:57-66.

58. Kantomaa MT, Tammelin TH, Ebeling HE, Taanila AM. Emotional and behavioral problems in relation to physical activity in youth. Med Sci Sports Exerc. 2008;40:1749-1756.

59. Kantomaa MT, Stamatakis E, Kankaanpää A, et al. Physical activity and obesity mediate the association between childhood motor function and adolescents' academic achievement. Proc Nat Acad Sci. 2013;110:1917-1922.

60. Rodriguez A, Jarvelin M-R, Obel C, et al. Do inattention and hyperactivity symptoms equal scholastic impairment? Evidence from three European cohorts. BMC Public Health. 2007;7:327.

61. Fergusson DM, Horwood LJ. Early conduct problems and later life opportunities. J Child Psychol Psychiatry. 1998;39:1097-1108. 